EPJ Web of Conferences 47, 13005 (2013)

DOI: $10.1051 /$ epjconf/20134713005

(C) Owned by the authors, published by EDP Sciences, 2013

\title{
Re-evaluating hot Jupiter WASP-12b: An update
}

\author{
Ian J.M. Crossfield ${ }^{1, a}$, Travis Barman², Brad M.S. Hansen ${ }^{3}$, Ichi Tanaka ${ }^{4}$ \\ and Tadayuki Kodama ${ }^{4}$ \\ ${ }^{1}$ Max-Planck-Institut für Astronomie, Königstuhl 17, 69117 Heidelberg, Germany \\ ${ }^{2}$ Lowell Observatory, 1400 West Mars Hill Road, Flagstaff, AZ 86001, USA \\ ${ }^{3}$ Department of Physics \& Astronomy, University of California Los Angeles, Los Angeles, \\ CA 90095, USA \\ ${ }^{4}$ Subaru Telescope, National Astronomical Observatory of Japan, 650 North A'ohoku Place, \\ Hilo, HI 96720, USA
}

\begin{abstract}
The hot Jupiter WASP-12b is one of the largest, hottest, and best-studied extrasolar planets. We revisit our recent analysis of WASP-12b's emission spectrum in light of near-infrared spectroscopic measurements which have been claimed to support either a hydride-dominated or carbon-rich atmospheric composition. We show that this new spectrum is still consistent with a featureless blackbody, indicating a nearly isothermal photosphere on the planet's day side. Thus the ensemble of occultation measurements for WASP-12b is still insufficient to constrain the planet's atmospheric composition.
\end{abstract}

\section{INTRODUCTION}

Although hot Jupiters occur less frequently around sun-like stars than do less massive planets [1, 2], these larger objects remain the most commonly known type of transiting planet ${ }^{1}$ because of the limiting sensitivity of current ground-based surveys. In addition, the high temperatures and large radii of hot Jupiters make these planets especially favorable targets for atmospheric characterization via transits $[3,4]$, secondary eclipses [5, 6], and phase curves [7, 8]. Thus the population of exoplanets for which atmospheric measurements have been made or attempted is largely limited to hot Jupiters.

The hot Jupiter WASP-12b is one of the largest and hottest transiting planets known [9-11]. The planet is intensely irradiated by its host star, which gives it an especially favorable planet/star flux contrast ratio. These conditions have motivated a flurry of photometric and spectroscopic occultation measurements [12-19]. Analysis of the earlier observations indicated an unusual atmospheric carbon to oxygen $(\mathrm{C} / \mathrm{O})$ ratio greater than one $[20]$.

However, the validity of the high $\mathrm{C} / \mathrm{O}$ conclusion is challenged by new $4.5 \mu \mathrm{m}$ data [14], the discovery of an M dwarf only $1^{\prime \prime}$ from WASP-12 which has contaminated past measurements [21], and our new $2.315 \mu \mathrm{m}$ narrowband occultation measurement [15]. Furthermore, a new controversy has arisen: recent 1-1.7 $\mu \mathrm{m}$ spectroscopy has been claimed to either support the high $\mathrm{C} / \mathrm{O}$ model [22] or to be inconsistent with it and to instead indicate a hydride-dominated atmosphere [19].

\footnotetext{
ae-mail: ianc@mpia.de

1 As of 31 Dec 2012: http://www . exoplanet. eu and http: /www . exoplanets.org

This is an Open Access article distributed under the terms of the Creative Commons Attribution License 2.0, which permits unrestricted use, distribution, and reproduction in any medium, provided the original work is properly cited.
} 


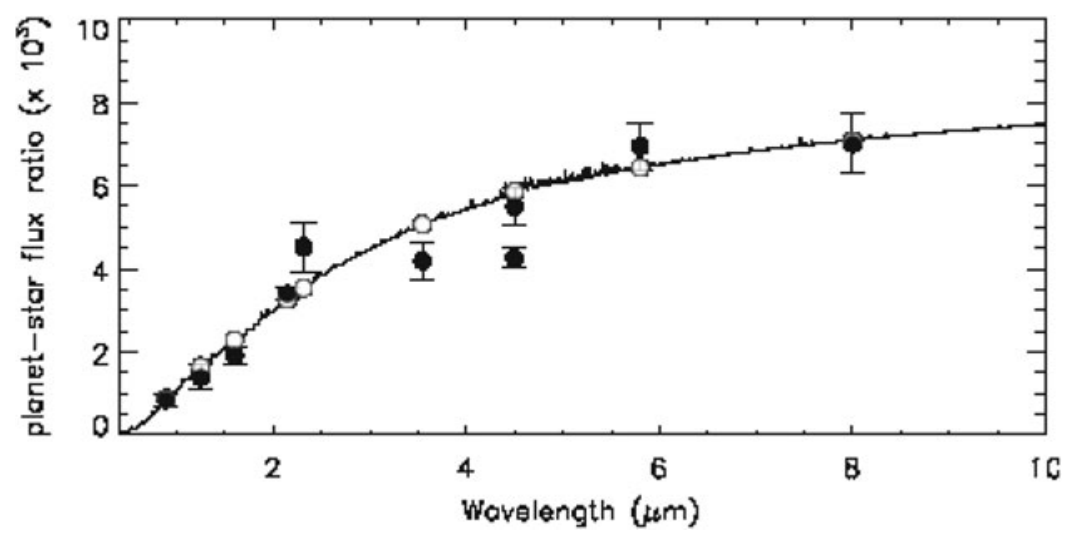

Figure 1. WASP-12b broad band emission spectrum (from [15]). Solid points are dilution-corrected photometric secondary eclipse depths; at $4.5 \mu \mathrm{m}$ we plot the two discrepant eclipse depths [13, 14]. The solid line shows the best-fit blackbody model; open symbols show band-integrated model values.

\section{WASP-12B'S EMISSION SPECTRUM}

\subsection{Photometric measurements}

As noted above, many secondary eclipses of WASP-12b have been observed using broadband photometry; the measurements span from $0.8-8 \mu \mathrm{m}[12-14,16,17]$. Most of these measurements are consistent with the high C/O interpretation, with one important exception. An analysis of WASP-12b's $4.5 \mu \mathrm{m}$ phase curve and eclipses [14] reported two possible conclusions: either the planet's secondary eclipse is significantly deeper at this wavelength than previously reported [13], or the occultations are consistent but the planet is extremely prolate (with an aspect ratio of 1.8). The latter possibility has been ruled out by recent WFC3 occultation spectroscopy ([19]; see also below), so the planet must be brighter at $4.5 \mu \mathrm{m}$ than previously thought.

In addition, our recent $2.315 \mu \mathrm{m}$ narrowband photometry [15] showed that the planet is also much brighter at this wavelength (by $>3 \sigma$ ) than high $\mathrm{C} / \mathrm{O}$ models predict [20]. We show the full photometric emission spectrum of WASP-12b in Fig. 1 (after correcting for Bergfors-6's dilution; see [15]). The impression given is that of a blackbody-like spectrum with few or no strong features. A simple blackbody model (representing a nearly isothermal dayside photosphere) gives $\chi^{2}=25$ and $\mathrm{BIC}^{2}=29$. The initial model used to claim a high $\mathrm{C} / \mathrm{O}$ ratio gave $\chi^{2}=10$ and $\mathrm{BIC}=32$ [20]; these values increase by $\sim 20$ when including the $2.315 \mu \mathrm{m}$ and average $4.5 \mu \mathrm{m}$ measurements.

Thus WASP-12b's photometric emission spectrum can be adequately explained by a nearly isothermal photosphere. In this case, the data do not justify claims of a high $\mathrm{C} / \mathrm{O}$ ratio in WASP$12 \mathrm{~b}$ 's atmosphere. We note here that a third $4.5 \mu \mathrm{m}$ occultation has been observed, which is more consistent with the shallow measurement than the deeper result (J. Harrington, private communication); if confirmed, this would have important repercussions for the interpretation of the planet's spectrum. A thorough and homogeneous analysis of all available WASP-12b occultations would be of great utility.

\subsection{Near-infrared 1-1.7 $\mu \mathrm{m}$ spectroscopy}

Recently, high-precision spectroscopic observations of WASP-12b's NIR emission have been obtained with HST/WFC3 [19]. This analysis notes that the initial C-rich model can match "the slope, but

\footnotetext{
${ }^{2}$ Bayesian Information Criterion (BIC) $=\chi^{2}+k \ln N$, where $k$ is the number of free parameters and $N$ the number of data points. When comparing two models to a data set, the model giving the lower BIC is statistically preferred.
} 


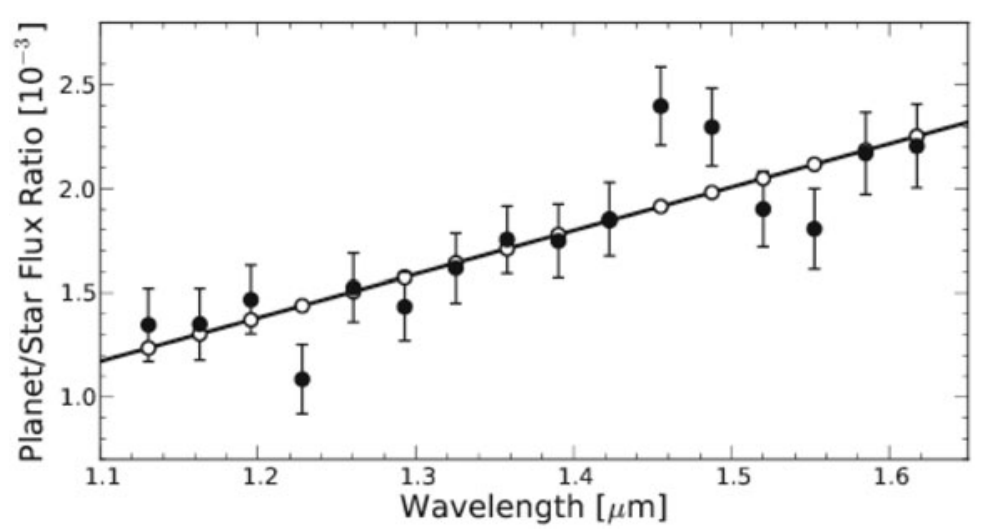

Figure 2. WASP-12b spectroscopic emission spectrum. Solid points show the HST/WFC3 spectroscopic measurements [19] after correction for Bergfors-6's dilution [15]. The solid line shows the weighted best-fit linear model; open symbols show band-integrated model values.

not the modulation" of their new near-infrared spectrum and that "the detailed shape of the emission spectrum [cannot] be modeled using $\mathrm{CO} / \mathrm{CO}_{2} / \mathrm{CH}_{4}$ opacity." Instead, the authors suggest that an atmosphere with a significant hydride complement better explains the NIR spectrum [19]. However, an independent reanalysis of this emission spectrum states that the authors of the spectroscopic analysis above "suggested that ... models with $\mathrm{C} / \mathrm{O} \geq 1$ best explained their data" [22], which seems to contradict the previously quoted statement.

Both these analyses probably read too much into the available data. We correct the $1.0-1.7 \mu \mathrm{m}$ emission spectrum for eclipse dilution (following [15]) and show it in Fig. 2. We also show a simple weighted linear fit to the data, which gives $\chi^{2}=19$ and BIC $=25$. The hydride-dominated model gives $\chi^{2}$ of roughly 13 [19]; assuming $k=10$ this gives $\mathrm{BIC}=41$, significantly larger than for our simpler model. Similarly, the updated high $\mathrm{C} / \mathrm{O}$ model gives $\chi^{2}=22$ and $\mathrm{BIC}=50$ for these data [22]; even if this model were fit only to the new WFC3 data, such a fit must give BIC $>k \ln N=28$ and so would still not be justified over the featureless toy model shown in Fig. 2.

One conclusion does seem robust: WASP-12b's $1.0-1.7 \mu \mathrm{m}$ emission spectrum shows no evidence for emission or absorption by water $[19,22]$. This result can be attributed either to a paucity of water in the planet's dayside atmosphere or to a dayside photosphere which is nearly isothermal.

\section{CONCLUSIONS}

At present, no strong conclusions can be drawn about the composition of WASP-12b's atmosphere on the basis of dayside (occultation) emission measurements. If future occultation measurements robustly detect strong emission or absorption by any species, this result would indicate a non-isothermal dayside photosphere; the lack of water features in WASP-12b's spectrum could then be attributed to a high $\mathrm{C} / \mathrm{O}$ ratio. The detection of only weak (or no) spectral features would be consistent with a roughly isothermal dayside photosphere, in which case secondary eclipse observations will be poorly suited to constrain the planet's atmospheric makeup. In this case, observations during primary transit may offer the best opportunity to determine the atmospheric composition of this unusual planet.

\section{References}

[1] A.W. Howard, G.W. Marcy, J.A. Johnson, D.A. Fischer, J.T. Wright, H. Isaacson, J.A. Valenti, J. Anderson, D.N.C. Lin, S. Ida, Science 330, 653 (2010), 1011.0143 
[2] A.W. Howard, G.W. Marcy, S.T. Bryson, J.M. Jenkins, J.F. Rowe, N.M. Batalha, W.J. Borucki, D.G. Koch, E.W. Dunham, T.N. Gautier, III et al., ApJS 201, 15 (2012), 1103. 2541

[3] G.W. Henry, G.W. Marcy, R.P. Butler, S.S. Vogt, ApJ 529, L41 (2000)

[4] D. Charbonneau, T.M. Brown, D.W. Latham, M. Mayor, ApJ 529, L45 (2000), arXiv: astro-ph/9911436

[5] D. Charbonneau, L.E. Allen, S.T. Megeath, G. Torres, R. Alonso, T.M. Brown, R.L. Gilliland, D.W. Latham, G. Mandushev, F.T. O’Donovan et al., ApJ 626, 523 (2005), arXiv:astro-ph/0503457

[6] D. Deming, T.M. Brown, D. Charbonneau, J. Harrington, L.J. Richardson, ApJ 622, 1149 (2005), arXiv: astro-ph/0412436

[7] J. Harrington, B.M. Hansen, S.H. Luszcz, S. Seager, D. Deming, K. Menou, J. Cho, L.J. Richardson, Science 314, 623 (2006)

[8] H.A. Knutson, D. Charbonneau, L.E. Allen, J.J. Fortney, E. Agol, N.B. Cowan, A.P. Showman, C.S. Cooper, S.T. Megeath, Nature 447, 183 (2007), 0705.0993

[9] L. Hebb, A. Collier-Cameron, B. Loeillet, D. Pollacco, G. Hébrard, R.A. Street, F. Bouchy, H.C. Stempels, C. Moutou, E. Simpson et al., ApJ 693, 1920 (2009), 0812. 3240

[10] T. Chan, M. Ingemyr, J.N. Winn, M.J. Holman, R. Sanchis-Ojeda, G. Esquerdo, M. Everett, AJ 141, 179 (2011), 1103.3078

[11] G. Maciejewski, R. Errmann, S. Raetz, M. Seeliger, I. Spaleniak, R. Neuhäuser, A\&A 528, A65 (2011), 1102.2421

[12] B. Croll, D. Lafreniere, L. Albert, R. Jayawardhana, J.J. Fortney, N. Murray, AJ 141, 30 (2011), 1009. 0071

[13] C.J. Campo, J. Harrington, R.A. Hardy, K.B. Stevenson, S. Nymeyer, D. Ragozzine, N.B. Lust, D.R. Anderson, A. Collier-Cameron, J. Blecic et al., ApJ 727, 125 (2011), 1003. 2763

[14] N.B. Cowan, P. Machalek, B. Croll, L.M. Shekhtman, A. Burrows, D. Deming, T. Greene, J.L. Hora, ApJ 747, 82 (2012), 1112.0574

[15] I.J.M. Crossfield, T. Barman, B.M.S. Hansen, I. Tanaka, T. Kodama, ApJ 760, 140 (2012), 1210.4836

[16] M. López-Morales, J.L. Coughlin, D.K. Sing, A. Burrows, D. Apai, J.C. Rogers, D.S. Spiegel, E.R. Adams, ApJ 716, L36 (2010), 0912.2359

[17] M. Zhao, J.D. Monnier, M.R. Swain, T. Barman, S. Hinkley, ApJ 744, 122 (2012), 1109. 5179

[18] I.J.M. Crossfield, B.M.S. Hansen, T. Barman, ApJ 746, 46 (2012), 1201.1023

[19] M. Swain, P. Deroo, G. Tinetti, M. Hollis, M. Tessenyi, M. Line, H. Kawahara, Y. Fujii, A. Showman, S. Yurchenko, arXiv.org astro-ph.EP (2012), 1205.4736

[20] N. Madhusudhan, J. Harrington, K.B. Stevenson, S. Nymeyer, C.J. Campo, P.J. Wheatley, D. Deming, J. Blecic, R.A. Hardy, N.B. Lust et al., Nature 469, 64 (2011), 1012. 1603

[21] C. Bergfors, W. Brandner, S. Daemgen, B. Biller, S. Hippler, M. Janson, N. Kudryavtseva, K. Geißler, T. Henning, R. Köhler, MNRAS 428, 182 (2013), 1209. 4087

[22] N. Madhusudhan, ApJ 758, 36 (2012), 1209. 2412 